\title{
Synthesis and Characterization of Monodisperse Magnetite Hollow Microspheres
}

\author{
Francisco Márquez ${ }^{1 *}$, Teresa Campo ${ }^{2}$, María Cotto ${ }^{1}$, Ramón Polanco ${ }^{1}$, Rolando Roque ${ }^{1}$, Pedro Fierro $^{3}$, \\ José María Sanz ${ }^{2}$, Eduardo Elizalde ${ }^{2}$, Carmen Morant ${ }^{2}$ \\ ${ }^{1}$ School of Science and Technology, University of Turabo, USA; ${ }^{2}$ Departamento de Física Aplicada C-XII, Universidad Autónoma \\ de Madrid, Cantoblanco, Madrid, Spain; ${ }^{3}$ Department of Chemistry, University of Puerto Rico, Mayagüez, USA. \\ Email: fmarquez@suagm.edu
}

Received January $29^{\text {th }}, 2011$; revised February $15^{\text {th }}, 2011$; accepted March $3^{\text {rd }}, 2011$.

\begin{abstract}
Monodisperse hollow magnetite microspheres were successfully synthesized by an one-step process through a template-free hydrothermal approach employing simultaneously a mixture of $\mathrm{FeCl}_{3} \cdot 6 \mathrm{H}_{2} \mathrm{O}$ and ferrocene as precursor and a propylene glycol-isopropanol mixture as solvent. The morphologies and properties of the magnetite microspheres were characterized by X-ray diffraction (XRD), Raman and infrared spectroscopy, field emission scanning electron microscopy (FE-SEM), X-ray photoelectron spectroscopy (XPS), adsorption of nitrogen (BET method) and SQUID magnetometry. The results show that the microspheres consist of a single crystalline phase of magnetite. On the other hand, the size and the internal hollow of the microspheres depend on the temperature of growth and can be tuned by modifying the synthesis conditions.
\end{abstract}

Keywords: $\mathrm{Fe}_{3} \mathrm{O}_{4}$, Magnetite, Hollow Spheres

\section{Introduction}

During the last decade an increasing attention has been paid to the design and fabrication of nanomaterials with controlled shapes and morphologies. Recently, this research has been focused on the synthesis of functional materials with novel structures including nanorods, nanotubes, nanospheres or microspheres in a variety of sizes, ranging from a few tenths of nanometers to several hundreds of nanometers [1-4]. Among these materials, magnetic nanostructures [5-7] have attracted a great deal of attention due to their interesting advantages such as magnetic properties [8] or low toxicity $[9,10]$ and potential applications in different fields such as catalysis [11,12], information storage [13], ferrofluids [14], optoelectronics [15], spintronics [16], or even in biomedical fields as magnetic resonance imaging (MRI) $[17,18]$ or drugs delivery $[19,20]$. Magnetite $\left(\mathrm{Fe}_{3} \mathrm{O}_{4}\right)$ nanoparticles are among the most important magnetic materials and they have been prepared by different approaches such as sol-gel techniques [21], coprecipitation [22], ultrasound irradiation [23], hydrothermal methods [24,25], thermal decomposition of metalorganic compounds [26], and so on.
Among these magnetic materials, magnetite nanoparticles with an interior void, represent an interesting family of nanostructures with paramount relevance in biological applications. These materials are characterized by having a large surface area, a very low density and also a strong magnetic response that make them candidates for a number of potential applications. Thus, the internal hollow spaces may potentially be used as hosts for the encapsulation of guest molecules and sensitive compounds such as specific drugs, catalysts, highly toxic compounds or any other group of substances that in the living environment can be directed towards the desired objective by applying external magnetic fields $[27,28]$. Different strategies have been reported on the synthesis of monodisperse magnetite hollow particles such as the Kirkendall effect [29], molten salts [30], Ostwald ripening [31,32], using micelles as a template [33], layer by layer [34], and so on. These methods have in common the use of templates, including hard-templates (i.e. silica nanoparticles, polymer beads or carbon nanospheres) [35-37] and soft-templates (i.e. supramolecular micelles or vesicles, emulsion droplets and even gas bubbles) $[38,39]$. However, due to the difficulties in controlling 
the size when soft-templates are used, hollow spheres are normally synthesized by the hard-template procedure. After synthesis, the template should be selectively removed by different methods (i.e. thermal decomposition, dissolution or etching) to finally obtain the hollow nanospheres. Although strategies that use templates have provided interesting results, the synthetic procedures are often very complex and tedious, resulting in low yields, high costs and even the by-products obtained after removal of the templates can contaminate the synthesized materials. In this regard, the development of new synthetic strategies necessarily involves the use of novel template-free routes to produce hollow magnetite nanoparticles with a total control of the dimensions of the internal void and the thickness of the walls.

Here, we report an one-step hydrothermal procedure without the use of surfactants or templates. In our case, we have used a mixture of $\mathrm{FeCl}_{3} \cdot 6 \mathrm{H}_{2} \mathrm{O}$ and ferrocene as precursors and a propylene glycol-isopropanol mixture as solvent. One of the most interesting advantages of this method is the relatively low synthesis temperature used in some cases $\left(120^{\circ} \mathrm{C}\right)$ and also the possibility of controlling efficiently the dimensionality of the magnetite microspheres, including the thickness of the walls and the volume of the internal void. Thus, we have obtained hollow microspheres ranging from ca. 300 to $600 \mathrm{~nm}$ with diameters that can be adjusted by varying the synthesis temperature.

\section{Experimental Section}

\subsection{Synthesis of Hollow Microspheres}

All reagents used in the present investigation were of analytical grade. $\mathrm{FeCl}_{3} \cdot 6 \mathrm{H}_{2} \mathrm{O}$, propylene glycol and isopropanol were provided by Sigma-Aldrich and used as received, without further purification. Ferrocene (98\%) was purchased from Sigma-Aldrich and purified by double vacuum sublimation before use. MilliQ water (18.2 $\mathrm{M} \Omega \cdot \mathrm{cm}$ at $25^{\circ} \mathrm{C}$ ) was used for all experiments.

In a typical synthesis of hollow magnetite microspheres, $2 \mathrm{mmol}$ of ferrocene were added to $50 \mathrm{~mL}$ of isopropanol. The mixture was stirred magnetically at room temperature for 1 hour. Next, $1 \mathrm{mmol}$ of $\mathrm{FeCl}_{3} \cdot \mathrm{H}_{2} \mathrm{O}$ was added to this solution, followed by dropwise addition of $10 \mathrm{~mL}$ of propylene glycol. After addition was complete, the mixture was refluxed for 2 hours. Next, the solution was allowed to cool and centrifuged at $1000 \mathrm{rpm}$ for $10 \mathrm{~min}$. The small sediment was discarded and the supernatant was incorporated into Teflon-lined stainless steel autoclaves of $50 \mathrm{~mL}$ capacity and maintained at different temperatures (ranging from 120 to $200^{\circ} \mathrm{C}$ ) for 10 hours. After cooling to room temperature, the black sediment is collected and washed from the impurities of the supernatant by adding isopropanol and centrifuged at least 5 times. Next, the black powder was dried overnight at $70^{\circ} \mathrm{C}$ and maintained in sealed containers before characterization.

With the aim of modifying the microspheres size (including the wall thickness and void diameter) and size distribution of the nanoparticles, the influence of the reaction time, growth temperature and the initial reaction mixture was investigated.

\subsection{Characterization Methods}

Field emission scanning electron microscopy (FESEM) images were obtained using a JEOL JM-6400 microscope. Raman spectra were recorded using a Confocal Raman Microscope (Renishaw RM2000) equipped with laser sources at $514 \mathrm{~nm}$ and $633 \mathrm{~nm}$ and an electrically refrigerated CCD camera. The spectral resolution was set at $4 \mathrm{~cm}^{-1}$, laser power employed was less $15 \mathrm{~mW}$ and acquisition time of each spectrum was around $200 \mathrm{sec}$.

XPS measurements were performed on an ESCALAB 220I-XL spectrometer, by using the non-monochromated $\mathrm{Mg} \mathrm{K}_{\alpha}(1253.6 \mathrm{eV})$ radiation of a twin-anode, operating at $20 \mathrm{~mA}$ and $12 \mathrm{kV}$ in the constant analyzer energy mode with a pass energy of $50 \mathrm{eV}$. Before spectra acquisition, the samples were degassed for 24 hours. The vacuum during spectra acquisition was better than $5 \times 10^{-9}$ mbar. In order to remove charging shifts and deal with Fermi edge coupling problems, binding energies were corrected using the peak of the $\mathrm{C}-(\mathrm{C}, \mathrm{H})$ component coming from contamination carbon (set to $284.6 \mathrm{eV}$ ).

The specific surface area, the pore volume and the pore size distribution (PSD) of the hollow magnetite microspheres, were measured using an Autosorb-1. With the help of the $N_{2}$ adsorption isotherms, the micropore volume, $W_{M P}\left[\mathrm{~cm}^{2} / \mathrm{g}\right]$ was measured using the $t$-plot method and the specific surface area, $S\left[\mathrm{~m}^{2} / \mathrm{g}\right]$, applying the BET method [40]. The beginning of the adsorption isotherm is used for this purpose, i.e.,: $0.00001<P / P_{o}<$ 0.02 for the $\mathrm{t}$-plot and $0.05<P / P_{o}<0.3$, for the BET plot, where, $P$, is the equilibrium adsorption pressure, and $P_{0}$, is the vapor pressure of the adsorbate at the temperature of the adsorption experiment. For the calculation of the pore size distribution (PSD), the Saito and Foley (S-F) method was employed [40].

Fourier transform-infrared (FT-IR) spectra were recorded in transmission mode on a Thermo Scientific Nicolet iS10 FTIR spectrometer using $\mathrm{KBr}$ wafers. The spectra were collected with a resolution of $4 \mathrm{~cm}^{-1}$ and were made 200 scans per sample.

XRD diffractograms were obtained using a Bruker D8 Advance system in Bragg-Brentano vertical goniometer configuration. The angular measurements $(\theta-2 \theta)$ were made with reproducibility of: \pm 0.0001 degree, applying 
steps of 0.05 degrees from 5 to 110 degrees. The X-ray radiation source was a ceramic $\mathrm{X}$-ray diffraction $\mathrm{Cu}$ anode tube type KFL C $2 \mathrm{~K}$ of $2.2 \mathrm{~kW}$, with long fine focus. A Ni filter was placed to eliminate the $\mathrm{Cu}-\mathrm{K}_{\beta}$ radiation.

The magnetic properties ( $M-H$ curves) of the magnetite samples were measured by using a Quantum Design MPMS XL-7 superconducting quantum interference device (SQUID) magnetometer at room temperature.

\section{Results and Discussion}

\subsection{Morphological Characterization}

Typical synthesis of hollow magnetite nanoparticles have been carried out by using a hydrothermal procedure. Different experimental parameters, including temperature and reaction time, were tested during the synthesis to determine the effects on the morphology and size of the nanoparticles. Apparently, only the reaction temperature could be correlated with relevant changes in the nanoparticles. The effect of different reaction times (ranging from $2 \mathrm{~h}$ to $20 \mathrm{~h}$ ) was studied and it was found that after 4 hours of reaction, the particles were almost formed. The average particle size does not seem to depend on the time of growth but nevertheless, the sphericity of these improved from 8 hours of reaction. For this reason, we fixed the reaction time to $10 \mathrm{~h}$. Another parameter studied was the growth temperature. It was found that the average size and the hole and wall dimensions of hollow magnetite particles clearly depended on the reaction temperature. Results are summarized on Table 1.

The SEM images of the as-synthesized samples are shown in Figure 1. As can be seen there, magnetite particles show high sphericity. In all cases, the particles have an inner hole whose dimensions vary with the reaction temperature. Sample a was obtained at low temperature $\left(120^{\circ} \mathrm{C}\right)$. In this case, the thickness of the wall, as determined by SEM, was apparently of ca. $50 \mathrm{~nm}$ with an average external diameter of ca. $295 \mathrm{~nm}$. The higher the synthesis temperature (samples b and c) the average particle diameter increases to ca. 396 and $500 \mathrm{~nm}$ respectively. The sample obtained at $200^{\circ} \mathrm{C} \mathrm{d}$ is characterized by having the best homogeneity. In this case, we could not observe any broken particle. In similar synthesis conditions (at temperatures ranging from 180 to $210^{\circ} \mathrm{C}$ and without ferrocene as precursor) we could obtain magnetite microspheres with unchanged wall thickness and for this reason we can presumably admit that the wall thickness of sample d should be similar to the sample c.

\subsection{XRD Characterization}

Figure 2 shows the XRD patterns of samples synthesized at different temperatures. As can be seen there, all pat-
Table 1. Size and composition of the as-synthesized samples as a function of the growth temperature.

\begin{tabular}{ccccc}
\hline Sample & $\mathrm{T}\left({ }^{\circ} \mathrm{C}\right)^{1}$ & $\begin{array}{c}\text { Ext. Size } \\
(\mathrm{nm})\end{array}$ & $\begin{array}{c}\text { Int. Hollow } \\
(\mathrm{nm})^{1}\end{array}$ & Composition \\
\hline $\mathrm{A}$ & 120 & 295 & 190 & $\mathrm{Fe}_{3} \mathrm{O}_{4}$ \\
B & 130 & 302 & 200 & $\mathrm{Fe}_{3} \mathrm{O}_{4}$ \\
$\mathrm{C}$ & 150 & 396 & 170 & $\mathrm{Fe}_{3} \mathrm{O}_{4}$ \\
D & 180 & 502 & 280 & $\mathrm{Fe}_{3} \mathrm{O}_{4}$ \\
E & 200 & 570 & n.d.* & $\mathrm{Fe}_{3} \mathrm{O}_{4}$ \\
\hline
\end{tabular}

${ }^{1}$ Average diameter of the hollow; *Not determined.

terns indicate the crystalline nature of the samples. All reflections have been indexed and their observed intensities are in very good agreement with the inverse cubic spinel structure of $\mathrm{Fe}_{3} \mathrm{O}_{4}$ (JCPDS 85-1436). However, the $\gamma-\mathrm{Fe}_{2} \mathrm{O}_{3}$ and $\mathrm{Fe}_{3} \mathrm{O}_{4}$ phases are very similar and for this reason, the possible presence of the $\gamma-\mathrm{Fe}_{2} \mathrm{O}_{3}$ phase cannot be excluded only from the results obtained by XRD. Nevertheless, the unambiguously characterization of the phase can be assigned by XPS.

\subsection{XPS Characterization}

With the aim to distinguish between $\gamma-\mathrm{Fe}_{2} \mathrm{O}_{3}$ and $\mathrm{Fe}_{3} \mathrm{O}_{4}$ phases and clearly indentify the chemical composition, the surface structure was further characterized by XPS. Figure 3 shows the $\mathrm{Fe} 2 \mathrm{p}$ and $\mathrm{O} 1 \mathrm{~s}$ regions, corresponding to nanoparticles synthesized at different temperatures.

As can be seen in Figure 3(A), in all analyzed samples the main peaks appear at ca. 711.1 and $724.6 \mathrm{eV}$ indicating that the $\mathrm{Fe}$ is present as $\mathrm{Fe}_{3} \mathrm{O}_{4}$. As shown in Figure 3(B), the XPS profile corresponding to the O1s region is characterized by only one peak at ca. $531.1 \mathrm{eV}$ that can be attributed to O-Fe in magnetite phase. These assignations agree with the literature [41,42] and unambiguously evidence that the only phase present in the samples is $\mathrm{Fe}_{3} \mathrm{O}_{4}$. Apparently, the synthesis temperature affects only to the dimensions of the magnetite nanoparticles and not to the chemical composition and crystalline structure.

It is worth noting that the $\mathrm{Fe} / \mathrm{O}$ atomic ratio (as determined by XPS) for samples grown at different temperatures, ranged from 0.71 to 0.82 . The expected value for magnetite is 0.75 , being these results in agreement with the previous phase assignation.

\subsection{Infrared and Raman Characterizations}

The FT-IR spectra of the magnetite nanoparticles are shown in Figure 4(A). In all cases, infrared spectra are characterized by a strong absorption band in the range of $583 \mathrm{~cm}^{-1}$ (synthesized at $120^{\circ} \mathrm{C}$ ) to $570 \mathrm{~cm}^{-1}$ (synthesized at $200^{\circ} \mathrm{C}$ ). The observed shift is gradual and appears to 


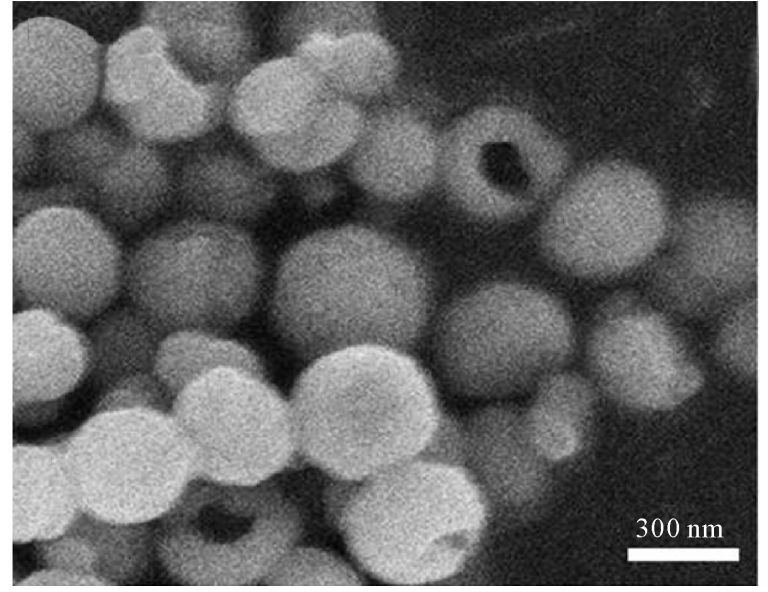

(a)

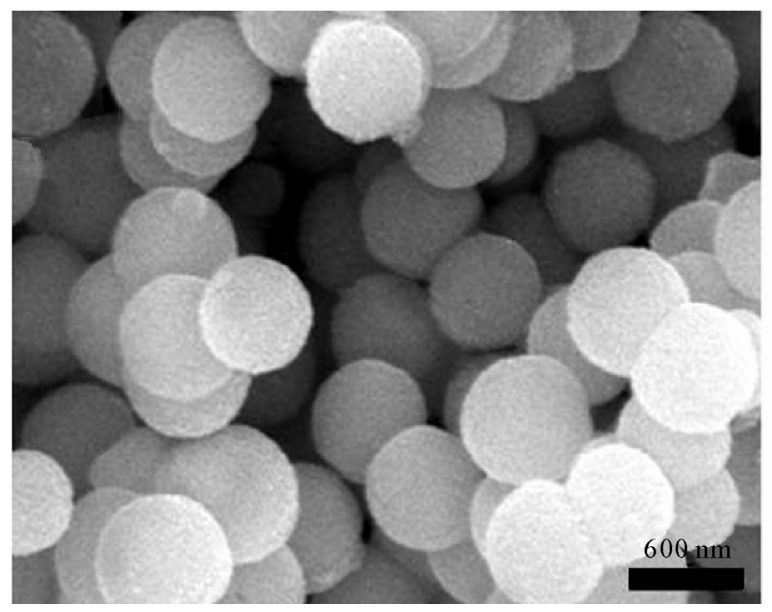

(c)

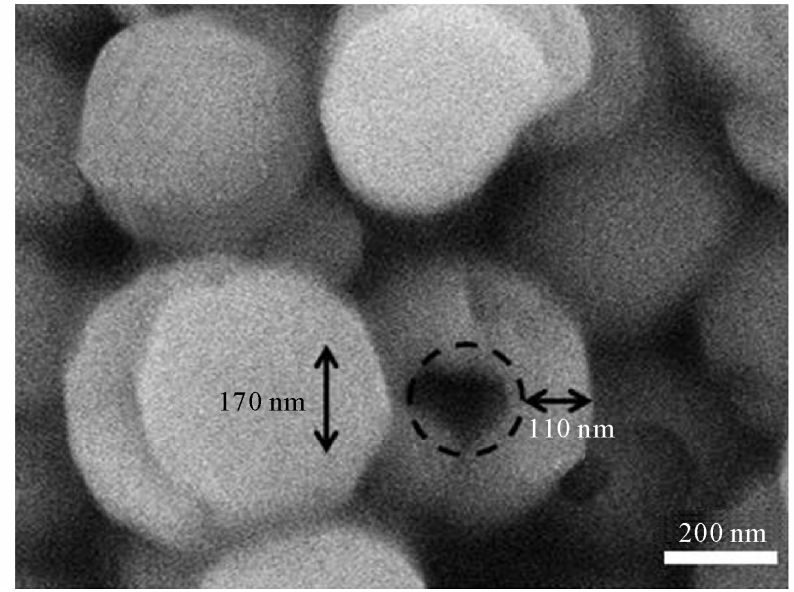

(b)

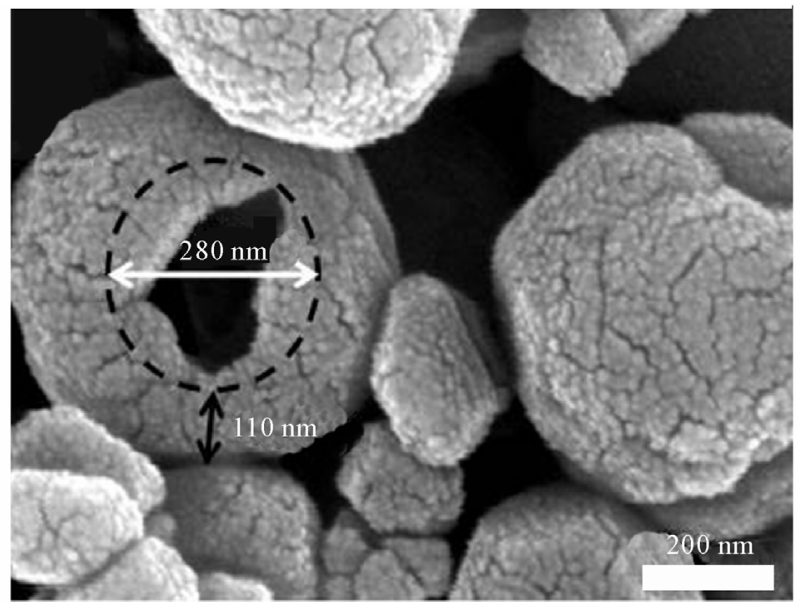

(d)

Figure 1. SEM images of the as-synthesized samples obtained at different temperatures: (a) $120^{\circ} \mathrm{C}$; (b) $150^{\circ} \mathrm{C}$; (c) $180^{\circ} \mathrm{C}$; (d) $200^{\circ} \mathrm{C}$.

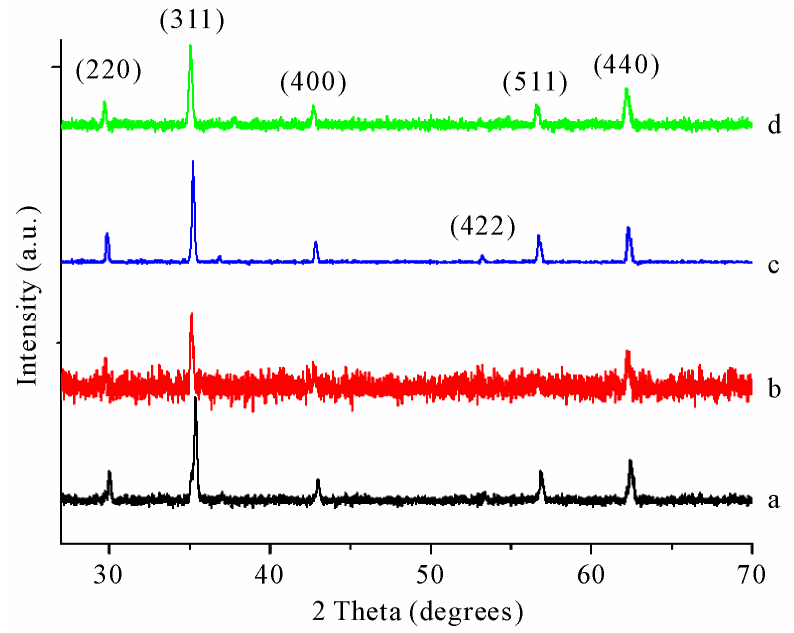

Figure 2. XRD patterns of the magnetite nanoparticles synthesized at different temperatures: (a) $120^{\circ} \mathrm{C}$; (b) $150^{\circ} \mathrm{C}$; (c) $180^{\circ} \mathrm{C}$; (d) $200^{\circ} \mathrm{C}$. depend on the particle size. Although this shift was unexpected, this main peak has been attributed to $\mathrm{Fe}-\mathrm{O} /$ $\mathrm{Fe}-\mathrm{O}-\mathrm{Fe}$ bindings of magnetite.

The structural phase of the synthesized nanoparticles has also been studied by Raman spectroscopy. Magnetite is characterized by having a main band at ca. $669 \mathrm{~cm}^{-1}$ $\left(\mathrm{A}_{1 \mathrm{~g}}\right)$ whereas $\gamma-\mathrm{Fe}_{2} \mathrm{O}_{3}$ shows broad bands around 700 and $500 \mathrm{~cm}^{-1}$ [43-45]. Figure 4(B) illustrates the Raman spectra measured for the samples synthesized at different temperatures. The smaller particles synthesized at $120^{\circ} \mathrm{C}$ (Figure 4(B)-a) exhibit a main band centered at 668 $\mathrm{cm}^{-1}$ and peaks at ca. $540 \mathrm{~cm}^{-1}$ and $300 \mathrm{~cm}^{-1}$ that have been assigned to $A_{1 g}, T_{2 g}$ and $E_{g}$ vibrations of magnetite. As can be seen in Figure 4(B), two peaks (marked as arrows) are observed in samples a and b. In samples c and $d$ these small peaks almost disappeared. This effect could be justified as possibly due to structural transformations induced by laser irradiation. In samples $a$ and $b$ 


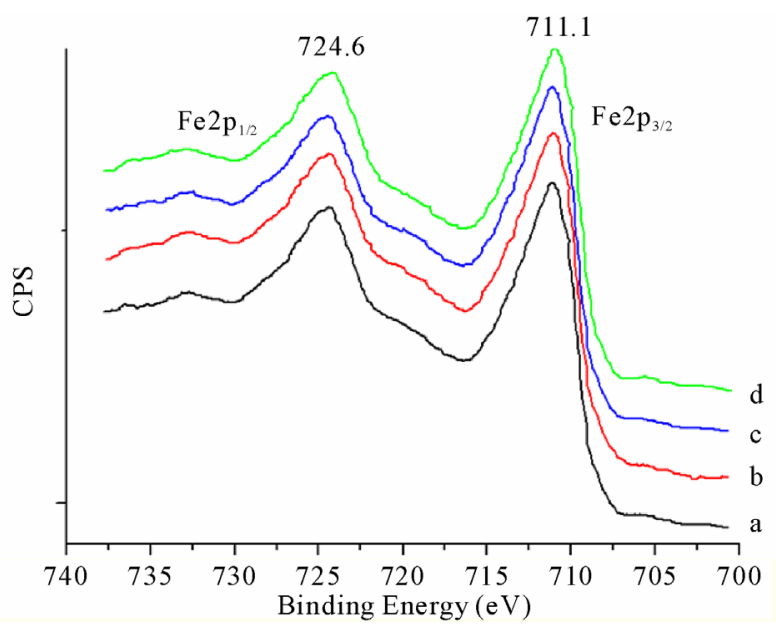

(A)

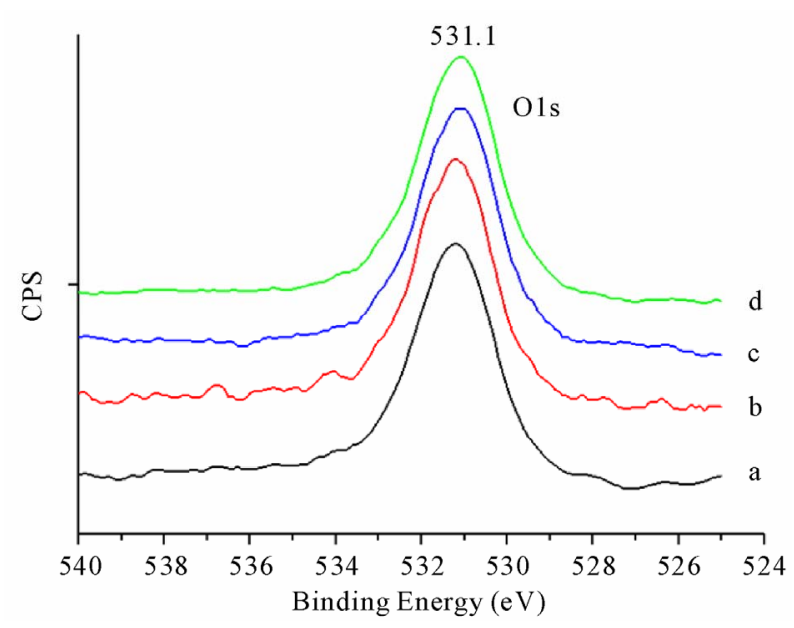

(B)

Figure 3. X-ray photoelectron spectroscopy of magnetite nanoparticles in the Fe2p (A) and O1s (B) regions, obtained at different synthesis temperatures: (a) $120^{\circ} \mathrm{C}$; (b) $150^{\circ} \mathrm{C}$; (c) $180^{\circ} \mathrm{C}$; (d) $200^{\circ} \mathrm{C}$.

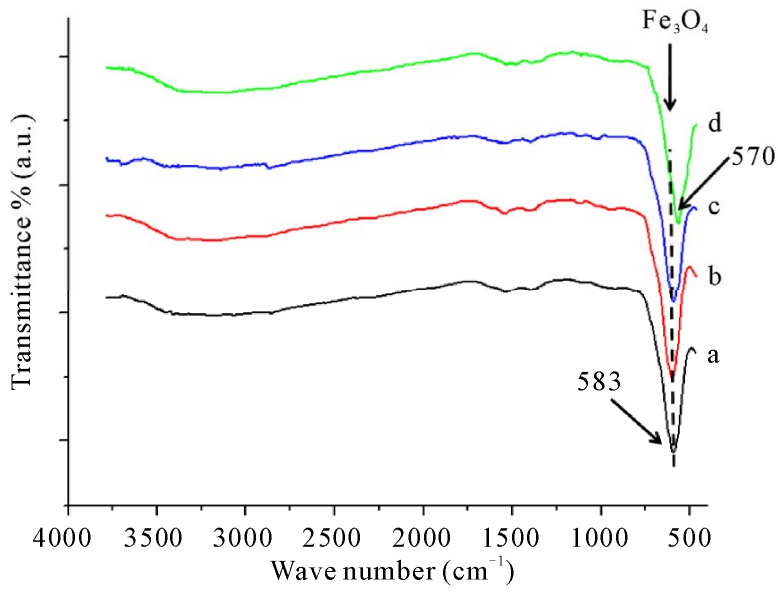

(A)

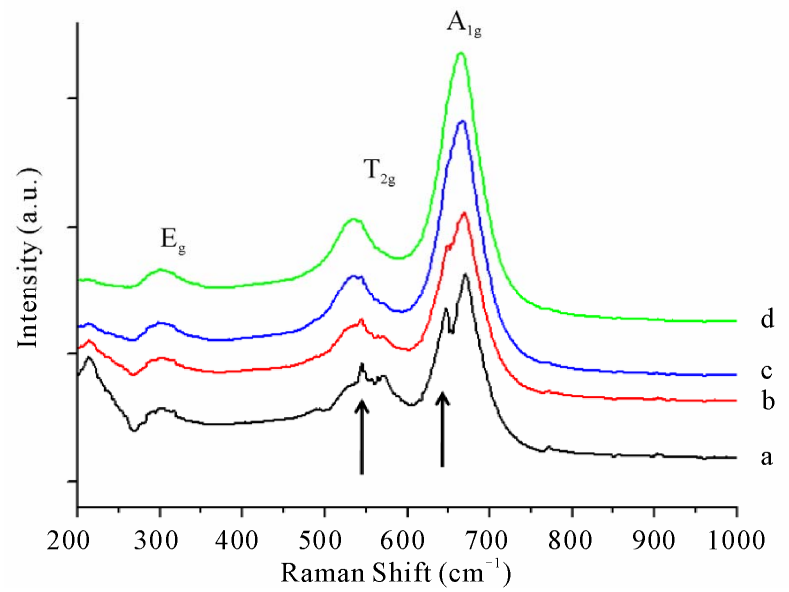

(B)

Figure 4. FT-IR (A) and Raman (B) spectra of the magnetite particles obtained at different synthesis temperatures: (a) $120^{\circ} \mathrm{C}$; (b) $150^{\circ} \mathrm{C}$; (c) $180^{\circ} \mathrm{C}$; (d) $200^{\circ} \mathrm{C}$.

the Raman signal was very low and it was necessary to increase the acquisition time, and subsequently the irradiation. This effect has been previously described in the literature [46] being even responsible for structural transformations from magnetite to maghemite.

\subsection{Magnetic Characterization}

Magnetic characterization of the hollow magnetite nanoparticles measured at $25^{\circ} \mathrm{C}$ is shown in Figure 5. As can be seen there, the analyzed samples exhibit ferromagnetic property at room temperature, with low coercivity values at 28.67 Oe (Figure 5(a)) and 60.50 Oe (Figure 5(b)). The saturation magnetization $\left(\mathrm{M}_{\mathrm{s}}\right)$ of sample synthesized at $200^{\circ} \mathrm{C}$ (Figure 5(a)) is highest at $25.65 \mathrm{emu} / \mathrm{g}$. This value is clearly higher than that observed for the sample synthesized at $120^{\circ} \mathrm{C}$ (Figure 5(b)) whose maximum $\mathrm{M}_{\mathrm{s}}$ is observed at $5.50 \mathrm{emu} / \mathrm{g}$. As determined by XPS, XRD and IR-Raman, in both cases, nanoparticles are composed by $\mathrm{Fe}_{3} \mathrm{O}_{4}$. The Ms values can be justified by the amount of magnetite in the sample that depends on the particle diameter and on the internal hollow. In fact, the Ms of the bulk magnetite is $92 \mathrm{emu} / \mathrm{g}$ [47] and, in our case, the highest value is observed for magnetite nanoparticles synthesized at $200^{\circ} \mathrm{C}$, with larger diameters $(600 \mathrm{~nm})$ and greater amount of magnetite. On the other hand, another possible effect that could be the reason for these $\mathrm{M}_{\mathrm{s}}$ values is the possible incorporation of metallic $\mathrm{Fe}$. In fact, the $\mathrm{Fe} / \mathrm{O}$ atomic ratio measured by XPS was higher in magnetite nanoparticles synthesized at $200^{\circ} \mathrm{C}$ (0.82) than in those synthesized at $120^{\circ} \mathrm{C}(0.70)$ being 


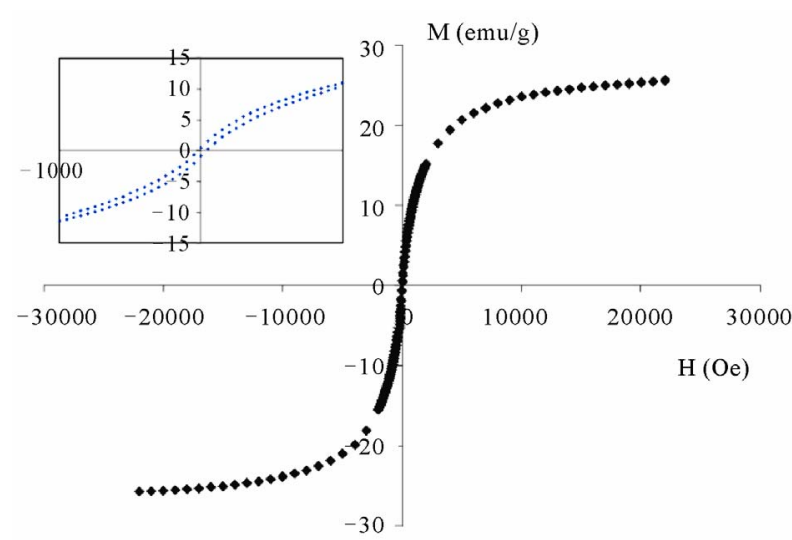

(a)

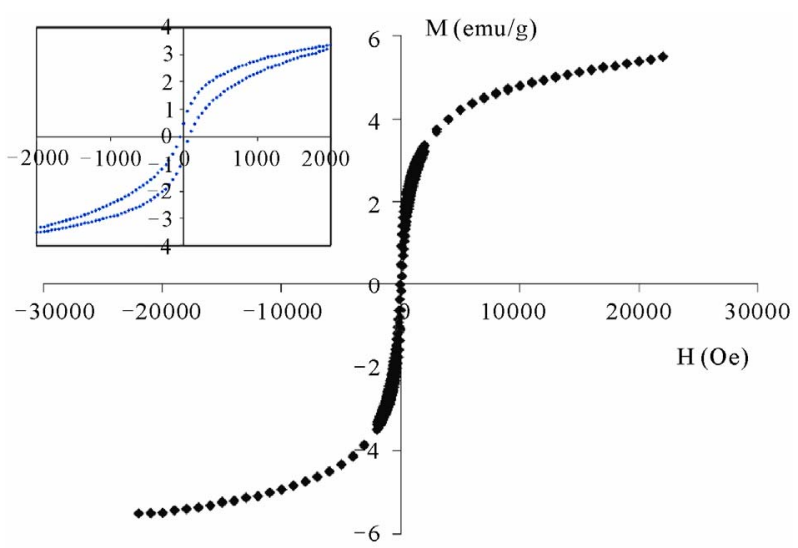

(b)

Figure 5. Magnetization curves obtained at $25^{\circ} \mathrm{C}$ of hollow magnetite nanoparticles synthesized at $200^{\circ} \mathrm{C}\left(\right.$ a) and $120^{\circ} \mathrm{C}$ (b). Insets in (a) and (b) correspond to magnified images of the origin region.

this result in agreement with the possible presence of $\mathrm{Fe}^{0}$ in the magnetite nanoparticles with larger diameters.

\subsection{Adsorption Isotherm}

The Brunauer-Emmett-Teller (BET) surface area and pore parameters of the sample synthesized at $120^{\circ} \mathrm{C}$ was determined by nitrogen adsorption-desorption isotherm measurement at $77 \mathrm{~K}$. As determined by SEM, the nanoparticle diameter of the samples synthesized at low temperature is ca. $300 \mathrm{~nm}$. In this case nanoparticles have a surface area of $60 \mathrm{~m}^{2} \mathrm{~g}^{-1}$ and an average pore diameter of $4 \mathrm{~nm}$. An explanation for these values could be found in the fact that nanoparticles are composed of much smaller primary nanoparticles that are organized to form hollow spheres allowing the existence of pores. With the aim to clarify this possibility, further adsorption studies on the magnetite particles synthesized in the range of 130 to $200^{\circ} \mathrm{C}$ are in progress.

\section{Conclusions}

In this study, we have explored the synthesis of hollow magnetite nanoparticles via a simple hydrothermal process. $\mathrm{Fe}_{3} \mathrm{O}_{4}$ magnetic nanoparticles were synthesized at different temperatures using $\mathrm{FeCl}_{3} \cdot 6 \mathrm{H}_{2} \mathrm{O}$ and ferrocene as precursors and a mixture of propylene glycol-isopropanol as solvent. As a result we have obtained hollow microspheres whose internal void space and wall thickness depend on the synthesis temperature. Additional work is currently being undertaken in order to understand more fully the growth mechanism. Considering the magnetic properties and the presence of an internal void space, these magnetite nanoparticles may have potential applications in catalysis, bioengineering and biological fields.

\section{Acknowledgements}

The authors gratefully recognize the financial support provided by MEC through the grants MAT2006-08158 and MAT2010-19804. Financial support from US Department of Energy through the Massey Chair project at University of Turabo and from the National Science Foundation under the project CHE-0959334 is also acknowledged. The "Servicio Interdepartamental de Investigación (SIdI)" from Universidad Autónoma de Madrid and "Centro de Microscopía Luis Bru" from Universidad Complutense de Madrid are acknowledged for the use of the FESEM facilities.

\section{REFERENCES}

[1] J. Liu and D. F. Xue, "Thermal Oxidation Strategy towards Porous Metal Oxide Hollow Architectures," Advanced Materials, Vol. 20, No. 13, 2008, pp. 2622-2627. doi:10.1002/adma.200800208

[2] Q. X. Guo, Y. S. Zhao, W. L. Mao, Z. W. Wang, Y. J. Xiong and Y. N. Xia, "Cubic to Tetragonal Phase Transformation in Cold-Compressed Pd Nanocubes," Nano Letters, Vol. 8, No. 3, 2008, pp. 972-975. doi:10.1021/n10731217

[3] C. L. Yang and D. F. Xue, "Formation of $\mathrm{Nb}_{2} \mathrm{O}_{5}$ Nanotube Arrays through Phase Transformation," Advanced Materials, Vol. 20, No. 5, 2008, pp. 1055-1058.

[4] H. Colfen and S. Mann, "Higher-Order Organization by Mesoscale Self-Assembly and Transformation of Hybrid Nanostructures," Angewandte Chemie International Edition, Vol. 42, No. 21, 2003, pp. 2350-2365. doi:10.1002/anie.200200562

[5] D. Yu, X. Sun, J. Zou, Z. Wang, F. Wang and K. Tang, "Oriented Assembly of Fe3O4 Nanoparticles into Monodisperse Hollow Single-Crystal Microspheres," Journal of Physical Chemistry B, Vol. 110, No. 43, 2006, pp. 21667-21671. doi:10.1021/jp0646933 
[6] H. Deng, X. Li, Q. Peng, X. Wang, J. Chen and Y. Li, "Monodisperse Magnetic Single-Crystal Ferrite Microspheres," Angewandte Chemie International Edition, Vol. 117, No. 18, 2005, pp. 2782-2785. doi:10.1002/anie.200462551

[7] A. H. Lu, E. L. Salabas and F. Schuth, "Magnetic Nanoparticles: Synthesis, Protection, Functionalization, and Application," Angewandte Chemie International Edition, Vol. 46, No. 8, 2007, pp.1222-1244. doi:10.1002/anie. 200602866

[8] J. Ge, Y. Hu, M. Biasini, W. P. Beyermann and Y. Yin, "Superparamagnetic Magnetite Colloidal Nanocrystal Clusters," Angewandte Chemie International Edition, Vol. 46, No. 23, 2007, pp. 4342-4345. doi:10.1002/anie.200700197

[9] D. Hogemann, V. Ntziachristos, L. Josephson and R. Weissleder, "High Throughput Magnetic Resonance Imaging for Evaluating Targeted Nanoparticle Probes," Bioconjugate Chemistry, Vol. 13, No. 1, 2002, pp. 116-121. doi: $10.1021 / \mathrm{bc} 015549 \mathrm{~h}$

[10] L. Gao, J. Wu, S. Lyle, K. Zehr, L. Cao and D. Gao, "Magnetite Nanoparticle-Linked Immunosorbent Assay," Jour- nal of Physical Chemistry C, Vol. 112, No. 44, 2008, pp. 17357- 17361. doi:10.1021/jp805994h

[11] G. P. Escobar, A. Q. Beroy, M. P. P. Iritia and J. H. Huerta, "Kinetic Study of the Combustion of Methyl-Ethyl Ketone over A-Hematite Catalyst," Chemical Engineering Journal, Vol. 102, No. 2, 2004, pp. 107-117.

[12] W. Werner and R. Wolfgang, "Surface Chemistry and Catalysis on Well-Defined Epitaxial Iron-Oxide Layers," Progress in Surface Science, Vol. 70, No. 1-3, 2002, pp. $1-151$.

[13] C. T. Black, C. B. Murray, R. L. Sandstrom, S. Sun, "Spin-Dependent Tunneling in Self-Assembled CobaltNano-crystal Superlattices," Science, Vol 290, No. 5494, 2000, pp. 1131-1134. doi:10.1126/science.290.5494.1131

[14] M. T. López-López, J. G. D. Durán, A. V. Delgado, F. J. González-Caballero, "Stability and Magnetic Characterization of Oleate-Covered Magnetite Ferrofluids in Different Nonpolar Carriers," Journal of Colloid and Interface Science, Vol. 291, No. 1, 2005, pp. 144-151.

[15] H. Zeng, J. Li, J. P. Liu, Z. L. Wang, S. H. Sun, "Exchange-Coupled Nanocomposite Magnets by Nanoparticle Self-Assembly," Nature, Vol. 420, No. 6914, 2002, pp. 395-398. doi:10.1038/nature01208

[16] S. Tiwari, R. J. Choudhary and D. M. Phase, "Effect of Growth Temperature on the Structural and Transport Properties of Magnetite Thin Films Prepared by Pulse Laser Deposition on Single Crystal Si Substrate," Thin Solid Films, Vol. 517, No. 11, 2009, pp. 3253-3256. doi:10.1016/j.tsf.2008.11.074

[17] M. S. Martina, J. P. Fortin, C. Menager, O. Clement, G. Barratt, C. Grabielle-Madelmont, F. Gazeau, V. Cabuil and S. Lesieur, "Generation of Superparamagnetic Liposomes Revealed as Highly Efficient MRI Contrast Agents for in Vivo Imaging," Journal of the American Chemical Society, Vol. 127, No. 30, 2005, pp. 10676-10685. doi: $10.1021 / \mathrm{ja} 0516460$

[18] N. A. Frey, S. Peng, K. Cheng and S. Sun, "Magnetic Nanoparticles: Synthesis, Functionalization, and Applications in Bioimaging and Magnetic Energy Storage," Chemistry Society Reviews, Vol. 38, No. 9, 2009, pp. 2532-2542. doi:10.1039/b815548h

[19] J. Dobson, "Magnetic Nanoparticles for Drug Delivery," Drugs Development Research, Vol. 67, No. 1, 2006, pp. 55-60. doi:10.1002/ddr.20067

[20] A. Schlachter, M. E. Gruner, M. Spasova, M. Farle and P. Entel, "Preparation and Properties of Nanostructured Magnetic Hollow Microspheres: Experiment and Simulation," Phase Transitions, Vol. 78, No. 9\&11, 2005, pp. 741-750. doi:10.1080/01411590500288809

[21] H. Itoh and T. Sugimoto, "Systematic Control of Size, Shape, Structure, and Magnetic Properties of Uniform Magnetite and Maghemite Particles," Journal of Colloid and Interface Science, Vol. 265, No. 2, 2003, pp. 283-295. doi:10.1016/S0021-9797(03)00511-3

[22] S. H. Gee, Y. K. Hong, D. W. Erickson and M. H. Park, "Synthesis and Aging Effect of Spherical Magnetite $\left(\mathrm{Fe}_{3} \mathrm{O}_{4}\right)$ Nanoparticles for Biosensor Applications," Journal of Applied Physics, Vol. 93, No. 10, 2003, pp. 7560-7562. doi:10.1063/1.1540177

[23] L. A. Harris, J. D. Goff, A. Y. Carmichael, J. S. Riffle, J. J. Harburn, T. G. St. Pierre and M. Saunders, "Magnetite Nanoparticle Dispersions Stabilized with Triblock Copolymers," Chemistry of Materials, Vol. 15, No. 6, 2003, pp. 1367-1377. doi:10.1021/cm020994n

[24] F. Márquez, C. Morant, J. M. Sanz and E. Elizalde, “Attachment of Magnetite Nanoparticles on Carbon Nanotubes Bundles and Their Response to Magnetic Fields," Journal of Nanoscience and Nanotechnology, Vol. 9, 2009, pp. 3810-3814. doi:10.1166/jnn.2009.NS72

[25] F. Márquez, C. Morant, J. M. Sanz and E. Elizalde, "Post-Synthesis Alignment of Chemically Modified Carbon Nanotubes in Magnetic Fields," Journal of Nanoscience and Nanotechnology, Vol. 9, 2009, pp. 61276131.

[26] J. L. Lyon, D. A. Fleming, M. B. Stone, P. Schiffer and M. E. Williams, "Synthesis of Fe Oxide Core/Au Shell Nano-particles by Iterative Hydroxylamine Seeding," Nano Letters, Vol. 4, No. 4, 2004, pp. 719-723. doi:10.1021/n1035253f

[27] S. Peng and S. Sun, "Synthesis and Characterization of Hollow $\mathrm{Fe}_{3} \mathrm{O}_{4}$ Nanoparticles," Angewandte Chemie International Edition, Vol. 46, No. 22, 2007, pp. 4155-4157. doi:10.1002/anie.200700677

[28] B. P. Jia and L. Gao, "Morphological Transformation of $\mathrm{Fe}_{3} \mathrm{O}_{4}$ Spherical Aggregates from Solid to Hollow and Their Self-Assembly under an External Magnetic Field," Journal of Physical Chemistry C, Vol. 112, No. 3, 2008, pp. 666-671. doi:10.1021/jp0763477

[29] H. J. Fan, U. Gösele and M. Zacharias, "Formation of Nanotubes and Hollow Nanoparticles Based on Kirken- 
dall and Diffusion Processes: A Review," Small, Vol. 3, No. 10, 2007, pp. 1660-1671.

doi:10.1002/smll.200700382

[30] D. Kim, J. Park, K. An, N. K. Yang, J. G. Park and T. Hyeon, "Synthesis of Hollow Iron Nanoframes," Journal of the American Chemical Society, Vol. 129, No. 18, 2007, pp. 5812- 5813. doi:10.1021/ja070667m

[31] P. Hu, L. Yu, A. Zuo, C. Guo and F. Yuan, "Fabrication of Monodisperse Magnetite Hollow Spheres," Journal of Physical Chemistry C, Vol. 113, No. 3, 2009, pp. 900-906. doi:10.1021/jp806406c

[32] X. Wang, F. L. Yuan, P. Hu, L. J. Yu and L. Bai, "Self-Assembled Growth of Hollow Spheres with Octahedron-like Co Nanocrystals via One-Pot Solution Fabrication," Journal of Physical Chemistry C, Vol. 112, No. 24, 2008, pp. 8773- 8778. doi:10.1021/jp0775404

[33] D. B. Yu, X. Q. Sun, J. W. Zou, Z. R. Wang, F. Wang and $\mathrm{K}$. Tang, "Oriented Assembly of $\mathrm{Fe}_{3} \mathrm{O}_{4}$ Nanoparticles into Monodisperse Hollow Single-Crystal Microspheres," Journal of Physical Chemistry B, Vol. 110, No. 43, 2006, pp. 21667-21671.doi:10.1021/jp0646933

[34] D. Lee, R. E. Cohen and M. F. Rubner, "Heterostructured Magnetic Nanotubes," Langmuir, Vol. 23, No. 1, 2007, pp. 123-129. doi:10.1021/1a0612926

[35] Q. L. Ye, Y. Kozuka, H. Yoshikawa, K. Awaga, S. Bandow and S. Iijima, "Effects of The Unique Shape of Submicron Magnetite Hollow Spheres on Magnetic Properties and Domain States," Physical Review B, Vol. 75, No. 22, 2007, pp. 224404-224408. doi:10.1103/PhysRevB.75.224404

[36] M. Ohnish, Y. Kozuka, Q. L. Ye, H. Yoshikawa, K. Awaga, R. Matsuno, M. Kobayashi, A. Takahara, T. Yokoyama, S. Bandow and S. Iijima, "Phase Selective Preparations and Surface Modifications of Spherical Hollow Nanomagnets," Journal of Material Chemistry, Vol. 16, No. 31, 2006, pp. 3215-3220. doi:10.1039/b605472b

[37] H. P. Liang, H. M. Zhang, J. S. Hu, Y. G. Guo, L. J. Wan and C. L. Bai, "Pt Hollow Nanospheres: Facile Synthesis and Enhanced Electrocatalysts," Angewandte Chemie International Edition, Vol. 43, No. 12, 2004, pp. 1540-1543. doi:10.1002/anie.200352956

[38] J. C. Bao, Y. Y. Liang, Z. Xu and L. Si, "Facile Synthesis of Hollow Nickel Submicrometer Spheres," Advanced Materials, Vol. 15, No. 21, 2003, pp. 1832-1835. doi:10.1002/adma.200305315

[39] L. T. Lu, L. D. Tung, J. Long, D. G. Fernig and N. T. K. Thanh, "Facile Synthesis of Stable, Water-Soluble Magnetic Copt Hollow Nanostructures Assisted by Multi- hiol Ligands," Journal of Material Chemistry, Vol. 19, No. 33, 2009, pp. 6023- 6028. doi:10.1039/b906839b

[40] A. Saito and H.C. Foley, "Curvature and Parametric Sensitivity in Models for Adsorption in Micropores," AIChE Journal, Vol. 37, No. 3, 1991, pp. 429-437. doi:10.1002/aic.690370312

[41] L. Y. Wang, J. Luo, Q. Fan, M. Suzuki, M. H. Engelhard, Y. Lin, N. Kim, J. Q. Wang and C. Zhong, "Monodispersed Core-Shell $\mathrm{Fe}_{3} \mathrm{O}_{4} @$ au Nanoparticles," Journal of Physical Chemistry B, Vol. 109, No. 46, 2005, pp. 21593-21601. doi:10.1021/jp0543429

[42] X. Teng, D. Black, N. Watkins, Y. Gao and H. Yang, "Platinum-Maghemite Core-Shell Nanoparticles Using a Sequential Synthesis," Nano Letters, Vol. 3, No. 2, 2003, pp. 261-264. doi:10.1021/n1025918y

[43] R. M. Cornell and U. Schwertmann, "The Iron Oxides," VCH, New York, 1996.

[44] O. N. Shebanova and P. J. Lazor, "Raman spectroscopic study of magnetite ( $\mathrm{FeFe} 2 \mathrm{O} 4)$ : a new assignment for the vibrational spectrum," Journal of Solid State Chemistry, Vol. 174, No. 2, 2003, pp. 424-430. doi:10.1016/S0022-4596(03)00294-9

[45] D. L. A. De Faria and S. Venancio Silva, M. T. De Oliveira, "Raman Microspectroscopy of Some Iron Oxides and Oxyhydroxides," Journal of Raman Spectroscopy, Vol. 28, No. 11, 1997, pp. 873-878. doi:10.1002/(SICI)1097-4555(199711)28:11<873::AID-J RS177>3.3.CO;2-2

[46] O. N. Shebanova and P. J. Lazor, "Raman Study of Magnetite $\left(\mathrm{Fe}_{3} \mathrm{O}_{4}\right)$ : Laser-Induced Thermal Effects and Oxidation," Journal of Raman Spectroscopy, Vol. 34, No. 11, 2003, pp. 845- 852. doi:10.1002/jrs. 1056

[47] X. Gao, K. M. K. Yu, K. Y. Tam and S. C. Tsang, "Colloidal Stable Silica Encapsulated Nano-Magnetic Composite as a Novel Bio-Catalyst Carrier," Chemical Communications, No. 24, 2003, pp. 2998-2999. doi: $10.1039 / \mathrm{b} 310435 \mathrm{~d}$ 